\title{
Avaliação clínica e radiográfica de pacientes operados pela técnica de Bristow-Latarjet com seguimento mínimo de 20 anos*
}

\section{Clinical and Radiographic Evaluation of Patients Operated by the Bristow-Latarjet Technique with a Minimum Follow-Up of 20 Years}

\author{
Jaime Guiotti Filho ${ }^{1}$ Marcelo Carvalho Leite ${ }^{1} \quad$ Antônio Carlos Wall Borges $^{1} \quad$ Gabriel Terra de Souza $^{1}$ \\ Otaniel Figueiredo do Prado ${ }^{1}$ \\ 1 Serviço de Ombro e Cotovelo, Instituto Ortopédico de Goiânia,
Goiânia, GO, Brasil \\ Rev Bras Ortop 2020;55(4):455-462. \\ Endereço para correspondência Jaime Guiotti Filho, Instituto \\ Ortopédico de Goiânia, Rua T-27, 819, setor Bueno, Goiânia, GO, \\ 74210-030, Brasil (e-mail: jaimeguiotti@gmail.com).
}

\section{Resumo}

Palavras-chave

- luxação do ombro

- instabilidade articular

- articulação do ombro

- estudos de seguimento
Objetivo Verificar os resultados do procedimento cirúrgico de 27 pacientes operados, durante o período de 1990 a 1997, pela técnica de Bristow-Latarjet para tratamento da instabilidade traumática anterior do ombro, considerando as possíveis complicações, e, principalmente, o aparecimento de artropatia.

Métodos A avaliação clínica subjetiva foi realizada por meio de um questionário respondido pelos pacientes, e a avaliação objetiva foi feita mediante o escore de Rowe et al. A avaliação radiográfica foi realizada usando as incidências anterioposterior (verdadeira), para visualizar a presença de sinais de artrose do ombro, segundo a classificação de Samilson e Prieto, e oblíqua apical e de Bernageau e Patte, para verificar a consolidação do enxerto ósseo, o posicionamento do parafuso, do enxerto, e os sinais de soltura do material de síntese. Estas avaliações foram realizadas por dois examinadores em tempos diferentes, sem interferência entre eles.

Resultados Na avaliação subjetiva dos pacientes, 93\% estavam totalmente recuperados, e, na avaliação objetiva, a média foi de 95 pontos na escala de Rowe et al. Não foram encontradas as complicações relativas à colocação do enxerto de coracoide. $\mathrm{O}$ grau de artropatia dos ombros, de acordo com a classificação de Samilson e Prieto, apresentou uma média de sete casos suaves, dois casos moderados, e um caso grave. No total, 17 pacientes não apresentaram artropatia.

Conclusão Entre a primeira e a segunda avaliações, não houve alteração quanto à eficácia da técnica de Bristow-Latarjet. A observação cuidadosa dos critérios da técnica foi fundamental para evitar complicações. A ocorrência de artropatia em longo prazo não foi relevante em nossa avaliação. Pelas evidências do presente estudo, somente o

Trabalho realizado no Centro de Estudos do Instituto Ortopédico de Goiânia, Goiânia, GO, Brasil.

recebido

06 de Setembro de 2018

aceito

12 de Março de 2019
DOI https://doi.org/

10.1055/s-0039-3402455. ISSN 0102-3616.
Copyright $\odot 2020$ by Sociedade Brasileira License terms de Ortopedia e Traumatologia. Published by Thieme Revinter Publicações Ltda, Rio de Janeiro, Brazil 


\section{Keywords}

- shoulder dislocation

- joint instability

- shoulder joint

- follow-up studies procedimento cirúrgico não é a causa do surgimento da artropatia, mas a falha na sua execução.
Objective To verify the results of 27 patients submitted to surgery from 1990 to 1997 by the Bristow-Latarjet technique for the treatment of anterior traumatic instability of the shoulder. The analysis included the possible complications, especially the appearance of arthropathy.

Methods The subjective clinical evaluation was performed through a questionnaire answered by the patients, and the objective evaluation was performed using the Rowe et al. score. The radiographic evaluation was performed using the anteroposterior (true) incidence to detect signs of shoulder arthrosis, according to the classification of Samilson and Prieto, as well as the apical oblique and the Bernageau and Patte incidences to verify the consolidation of the bone graft, the position of the screw and of the graft, and signs of the release of the synthesis material. These evaluations were performed by two examiners at different times without interference between them. Results In the subjective assessment of the patients, 93\% were fully recovered, and, in the objective evaluation, the average was 95 points on the Rowe et al. score. Complications related to coracoid placement were not found. The degree of arthropathy of the shoulders, according to the Samilson and Prieto classification, presented an average of seven mild cases, two moderate cases and one severe case. In total, 17 patients did not present arthropathy.

Conclusion Between the first and second evaluations, there was no change in the efficacy of the Bristow-Latarjet technique. The careful observation of the criteria of the technique was fundamental to avoid complications. The occurrence of arthropathy in the long term was not relevant in our evaluation. Based on the evidences of the present study, the surgical procedure alone is not the cause of the onset of the arthropathy, but the failure in its execution.

\section{Introdução}

A luxação anterior do ombro ocorre com grande frequência na população em geral, e principalmente nos praticantes de esportes de contato. ${ }^{1} \mathrm{~A}$ taxa de recorrência é maior em indivíduos com idade $\leq 20$ anos, e aproximadamente $50 \%^{2}$ são estabilizados cirurgicamente por várias técnicas. Uma das técnicas cirúrgicas empregadas para corrigir a instabilidade anterior do ombro é a cirurgia de Bristow-Latarjet. Existem pouco estudos de longo prazo sobre as complicações, como a recorrência da instabilidade, a colocação, a não consolidação, a quebra do parafuso de fixação do enxerto e, principalmente, a ocorrência de artropatia.

A instabilidade anterior do ombro foi por muito tempo corrigida somente por cirurgia aberta, até o surgimento da cirurgia artroscópica. Com a divulgação da cirurgia artroscópica e a evolução desta técnica, a cirurgia aberta passou a ser indicada para casos específicos, pela presença de lesão óssea na cabeça do úmero e na borda anterior da glenoide, conhecida hoje como lesão bipolar, que, na maioria das vezes, provoca maus resultados pela técnica artroscópica.

A cirurgia aberta mais usada é a transferência do processo coracoide, que foi inicialmente realizada por Bristow, como descrito por Helfet, ${ }^{2}$ colocando o enxerto sob o músculo subescapular. Em 1954, Latarjet ${ }^{3}$ descreveu a fixação do processo coracoide com um parafuso no colo da glenoide. Esta técnica foi modificada por Patte et al. ${ }^{4}$ que utilizaram uma parte maior do coracoide fixada no colo da glenoide com dois parafusos. May ${ }^{5}$ descreveu a colocação do processo coracoide com um parafuso.

Após a publicação de Helfet, ${ }^{2}$ a técnica cirúrgica aberta com transferência do processo coracoide para correção da instabilidade anterior do ombro passou a ser conhecida como cirurgia de Bristow-Latarjet. No Brasil, a técnica de BristowLatarjet foi amplamente divulgada por Ferreira Filho, ${ }^{6}$ pois foi objeto de estudo para sua tese de doutorado apresentada ao Departamento de Ortopedia da Faculdade de Medicina da Universidade de São Paulo em 1984.

Em 1987, a técnica de Bristow-Latarjet foi considerada não fisiológica por Young e Rockwood ${ }^{7}$ e também por outros autores, ${ }^{8-19}$ que defendiam a possibilidade de complicações resultantes da colocação incorreta, do deslocamento, e da quebra do parafuso, além de lesão neurovascular e artrite, reações que já seriam suficientes para a contraindicação desta técnica cirúrgica.

Weaver e Derkesh, ${ }^{20}$ em um estudo comparativo com a cirurgia de Bankart, não encontraram o alto índice de complicações atribuídas à cirurgia de Bristow-Latarjet. 
Em 1998, com o intuito de avaliar resultados e complicações da técnica cirúrgica, Guiotti Filho et al. ${ }^{21}$ avaliaram, durante 7 anos, 56 pacientes operados pela técnica de Bristow-Latarjet para tratamento da instabilidade traumática anterior do ombro, e concluíram pela eficiência da cirurgia, corroborada pela experiência acumulada.

Existem poucos estudos publicados com seguimento superior a 15 anos sobre a cirurgia de Bristow-Latarjet utilizada para instabilidade anterior do ombro. Gordins et al. ${ }^{22}$ fizeram um estudo comparativo das suas primeiras cirurgias de Bristow-Latarjet, com seguimento médio de 33 anos, o que nos motivou a rever nossos casos.

O objetivo deste trabalho foi realizar uma avaliação retrospectiva dos resultados e complicações da técnica de Bristow-Latarjet, com um seguimento mínimo de vinte anos, em pacientes operados pelo mesmo cirurgião. Para apresentar os dados comparativos, os 56 pacientes participantes do estudo de Guiotti Filho et al. ${ }^{21}$ foram convidados para uma nova avaliação entre 20 e 27 anos da data da cirurgia para verificar a atual situação do ombro acometido, considerando as possíveis complicações, e, principalmente, 0 aparecimento de artropatia.

\section{Materiais e Métodos}

Entre 1990 a 1997, 80 pacientes foram submetidos à cirurgia de Bristow-Latarjet, realizada pelo mesmo cirurgião, para o tratamento da luxação anterior traumática recorrente e unidirecional do ombro associada à lesão de Hill Sachs e à lesão óssea de Bankart. A cirurgia de Bristow-Latarjet foi indicada para os pacientes cujos exames radiográficos apresentassem evidências dessas lesões ósseas.

O procedimento cirúrgico de Bristow-Latarjet foi realizado por abordagem deltopeitoral, abertura do subescapular e cápsula em "L", para uma criteriosa colocação do enxerto de coracoide na posição vertical, com sua face inferior voltada para a cabeça do úmero, anterior e abaixo do equador da glenoide, sendo fixado com um parafuso paralelo à superfície articular, atingindo a cortical posterior e passando-a em $2 \mathrm{~mm}$. Desses 80 pacientes, 56 tiveram acompanhamento pós-cirúrgico entre o período de 1990 a 1997, e os resultados foram publicados por Guiotti Filho et al. ${ }^{21}$ não sendo encontradas, no curto prazo, complicações relativas ao enxerto e artrose.

Em continuidade ao estudo supracitado, o presente trabalho avaliou o pós-operatório com um seguimento mínimo de 20 anos da data da cirurgia destes mesmos pacientes, mas apenas 27 foram encontrados e aceitaram essa avaliação. Os 29 pacientes que não responderam ao nosso contato foram excluídos do estudo. $O$ objetivo foi verificar a condição atual desses 27 pacientes e avaliar o procedimento cirúrgico realizado à época. Todos 27 pacientes incluídos neste estudo estavam aptos para avaliação; 1 deles teve lesão axonal do nervo axilar antes da cirurgia realizada, e houve recuperação, mas ele evoluiu com atrofia do deltoide.

A avaliação objetiva ocorreu por meio de exame físico para verificação da amplitude de movimentos e da dor, e de testes da apreensão, e a avaliação subjetiva foi realizada por meio de um questionário respondido pelos pacientes (- Figura 1). A ava-

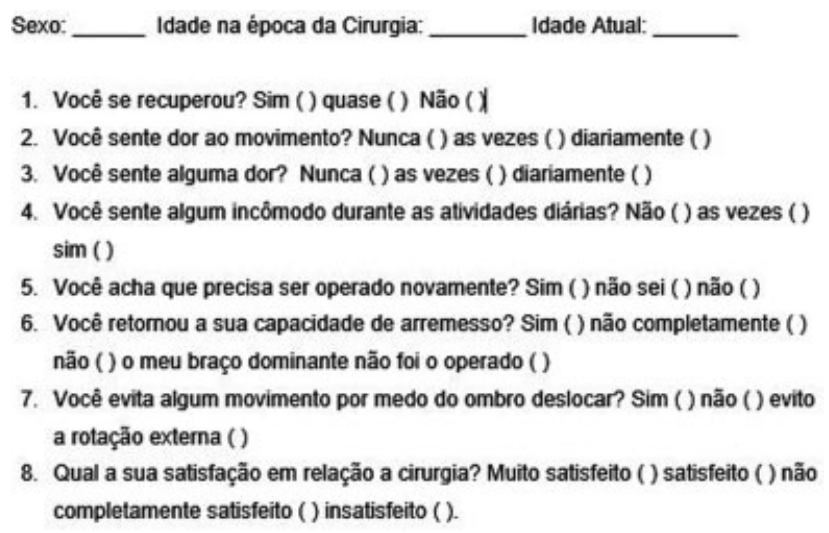

Sexo: Idade na época da Cirurgia: Idade Atual:

1. Você se recuperou? Sim ( ) quase ( ) Não ( $\mid$ X

2. Você sente dor ao movimento? Nunca ( ) as vezes () diariamente ()

3. Você sente alguma dor? Nunca ( ) as vezes ( ) diariamente ()

4. Você sente algum incômodo đurante as atividades diárias? Não ( ) as vezes ( ) $\operatorname{sim}()$

5. Você acha que precisa ser operado novamente? Sim ( ) não sei ( ) não ( )

6. Você retornou a sua capacidade de arremesso? Sim () não completamente () não ( ) o meu braço dominante não foi o operado ( )

7. Você evita algum movimento por medo do ombro deslocar? Sim ( ) não ( ) evito a rotação externa ()

8. Qual a sua satisfação em relação a cirurgia? Muito satisfeito ( ) satisfeito ( ) não completamente satisfeito () insatisfeito ().

Fig. 1 Instrumento de coleta - questionário subjetivo. Fonte: Gordins et al. 22

liação radiográfica, realizada para visualizar sinais de artrose, foi feita pela incidência anteroposterior (AP) (verdadeira), cujos graus foram determinados, segundo a classificação de Samilson e Prieto, ${ }^{23}$ como artrose leve (osteófito $<3 \mathrm{~mm}$ no úmero e na glenoide), artrose moderada (osteófito entre $3 \mathrm{~mm}$ e $7 \mathrm{~mm}$ no úmero e na glenoide), e artrose grave (osteófito $>8 \mathrm{~mm}$ no úmero e na glenoide, com diminuição do espaço articular e esclerose). A avaliação radiográfica para verificar a consolidação do enxerto ósseo, o posicionamento do parafuso e do enxerto, e os sinais de soltura do material de síntese foi feita usando as incidências de Bernageau e Patte ${ }^{24}$ e oblíqua apical. ${ }^{25}$ Estas avaliações foram realizadas somente no ombro operado por dois examinadores, identificados neste estudo como examinador 1 (E1) e examinador 2 (E2), em tempos diferentes e sem interferência entre eles.

As entrevistas com os pacientes aconteceram entre os meses de fevereiro e março de 2018, após aprovação do Comitê de Ética, sob o protocolo $n^{\circ} 2.383 .660$, diante da assinatura do paciente do termo de consentimento livre e esclarecido, e seguindo um protocolo de questionário subjetivo. A escala funcional de Rowe et al. ${ }^{17}$ (- Tabela 6) foi preenchida e avaliada no pós-operatório.

\section{Resultados}

O presente estudo avaliou 27 pacientes 20 anos após eles terem sido submetidos à cirurgia de Bristow-Latarjet. À época da cirurgia, os 27 pacientes tinham entre 17 e 59 anos, sendo $85 \%$ do sexo masculino e $15 \%$ feminino. A idade da primeira luxação variou entre 16 e 49 anos. 0 esporte praticado pela maioria (41\%) era o futebol, e o ombro acometido em $55 \%$ foi o do lado direito, e o esquerdo, em $45 \%$. 0 tempo médio desde a primeira luxação até a realização da cirurgia foi de 48 meses. Os participantes estão atualmente com idades entre 37 e 79 anos (- Tabela 1).

Na abordagem subjetiva, pelas respostas dos pacientes, 25 (93\%) foram considerados recuperados, e 2 (7\%), não totalmente recuperados. Dos 27 pacientes questionados sobre sentir dor, $4(15 \%)$ relataram sentir dor algumas vezes ao movimentar o braço acometido, e 2 (7\%) disseram sentir dor ao realizar atividades diárias. No total, 2 (7\%) gostariam de ter 
Tabela 1 Índice demográfico

\begin{tabular}{|c|c|c|}
\hline \multicolumn{3}{|c|}{ Sexo } \\
\hline & Gênero & Resultados \\
\hline & Masculino & 23 \\
\hline & Feminino & 4 \\
\hline \multicolumn{3}{|c|}{ Idade } \\
\hline & Média de idade dos pacientes (anos) & $52(37-74)$ \\
\hline & Faixa etária & Resultados \\
\hline & $\leq 20$ & 0 \\
\hline & 21 a 40 & 3 \\
\hline & 41 a 60 & 20 \\
\hline & 61 a 80 & 4 \\
\hline & $>80$ & 0 \\
\hline \multicolumn{3}{|c|}{ Idade na primeira luxação } \\
\hline & $\begin{array}{l}\text { Média de idade da primeira } \\
\text { luxação (anos) }\end{array}$ & $25(16-49)$ \\
\hline & Faixa etária & Resultados \\
\hline & $\leq 20$ & 8 \\
\hline & 21 a 40 & 17 \\
\hline & 41 a 60 & 2 \\
\hline & 61 a 80 & 0 \\
\hline & $>80$ & 0 \\
\hline \multicolumn{3}{|c|}{ Esporte praticado antes } \\
\hline & Esportes & Resultados \\
\hline & Musculação & 1 \\
\hline & Basquete & 1 \\
\hline & Capoeira & 1 \\
\hline & Futebol & 11 \\
\hline & Judô & 1 \\
\hline & Equitação & 1 \\
\hline & Natação & 4 \\
\hline & Nenhum & 6 \\
\hline & Não informado & 1 \\
\hline \multicolumn{3}{|c|}{ Lado acometido } \\
\hline & Direito & 15 \\
\hline & Esquerdo & 12 \\
\hline
\end{tabular}

uma recuperação melhor, 10 (37\%) relataram ter recuperado a capacidade de arremesso, e 2 (7\%) evitam alguns movimentos por medo de o ombro se deslocar. Ao serem questionados sobre a satisfação com os resultados após a cirurgia, 21 (78\%) consideram-se muito satisfeitos, 5 (18\%), satisfeitos, e 1 (4\%), não completamente satisfeito.

O grau de artropatia dos ombros, avaliado pelos dois examinadores (-Tabela 2 ) de acordo com a classificação de Samilson e Prieto, apresentou uma média de 7 casos suaves, 2 casos moderados, e 1 caso grave (-Figura 2 ), que teve seu primeiro episódio de luxação com mais de 22 anos de idade, e evoluiu com atrofia de músculo deltoide no longo
Tabela 2 Grau de artropatia segundo a classificação de Samilson e Prieto

\begin{tabular}{|l|l|l|l|}
\hline $\begin{array}{l}\text { Incidência } \\
\text { anteroposterior } \\
\text { (verdadeira) }\end{array}$ & Examinador 1 & Examinador 2 & Média \\
\hline Classificação & 17 & 17 & 17 \\
\hline Normal & 7 & 7 & 7 \\
\hline Suave (3 mm) & 7 & 2 & 2 \\
\hline $\begin{array}{l}\text { Moderada } \\
\text { (3-7 mm) }\end{array}$ & 2 & 1 & 1 \\
\hline Grave (>8 mm) & 1 & 17 & \\
\hline
\end{tabular}

Nota: Apenas um paciente apresentou artrose grave segundo a classificação. ${ }^{16}$

prazo. Esse paciente havia sido diagnosticado com lesão do nervo axilar pela eletroneuromiografia antes da cirurgia realizada, fato que pode ter colaborado para o desfecho de artrose grave. Os 17 pacientes restantes não apresentaram artropatia, como pode ser observado na - Figura 3.

A - Tabela 3 apresenta a correlação da idade com o grau de artropatia e com a restrição da rotação externa. Em 12 pacientes que tiveram a primeira luxação com menos de 22 anos, não foi observado nenhum caso de artropatia grave. Os 2 casos que apresentaram artropatia mais avançada foram considerados pelos 2 examinadores artropatia moderada, e ambos apresentaram restrição de rotação externa $>10^{\circ}$.

Dos 15 pacientes que luxaram o ombro com mais de 22 anos, 1 teve grau moderado de artropatia, com restrição da rotação externa $>10^{\circ}$.

Os 2 examinadores observaram 1 caso de artrose grave, com restrição da rotação externa $>15^{\circ}$.

Na avaliação objetiva e subjetiva ( - Tabela 4) dos movimentos, houve recuperação da elevação e da rotação externa do ombro na maioria dos pacientes (22; 84\%).

O sinal de apreensão tornou-se negativo na avaliação final em 24 (89\%) dos pacientes, não havendo recidiva da instabilidade. Dos pacientes com apreensão positiva, dois tinham quadros esporádicos de convulsão, e um sofreu fratura de origem traumática do material de síntese (-Figura 4).

Na avaliação radiológica nas incidências de Bernageaue Patte $^{24}$ e oblíqua apical ${ }^{25}$ ( - Figura 5), nenhum dos pacientes apresentou sinais de falta de consolidação do enxerto ósseo.

Em 1 paciente, o enxerto estava colocado mais medial em relação à superfície articular, situação avaliada pelo E2 (-Tabela 5), mas sem repercussão clínica.

Não houve diferença estatística entre as avaliações dos dois examinadores. A média da pontuação na análise da escala de Rowe et al. $^{17}$ (-Tabela 6) foi significativa: 95 pontos.

\section{Discussão}

Este estudo teve um seguimento médio de 24 anos, e incluiu todos os pacientes operados pelo procedimento de Bristow- 

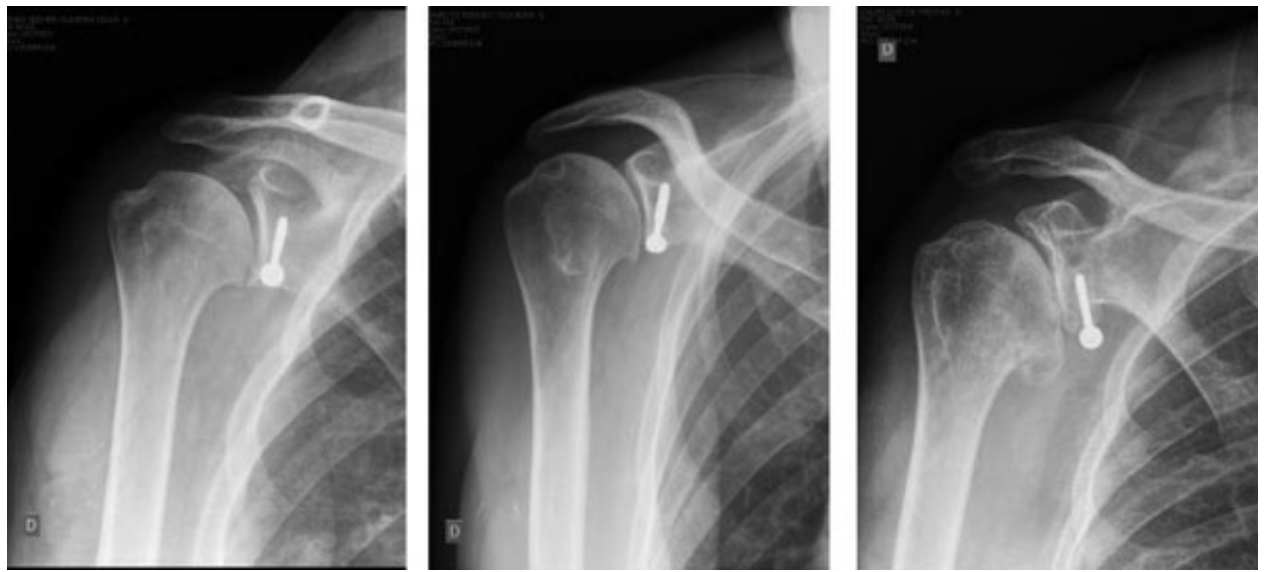

Fig. 2 Grau de artropatia dos ombros.

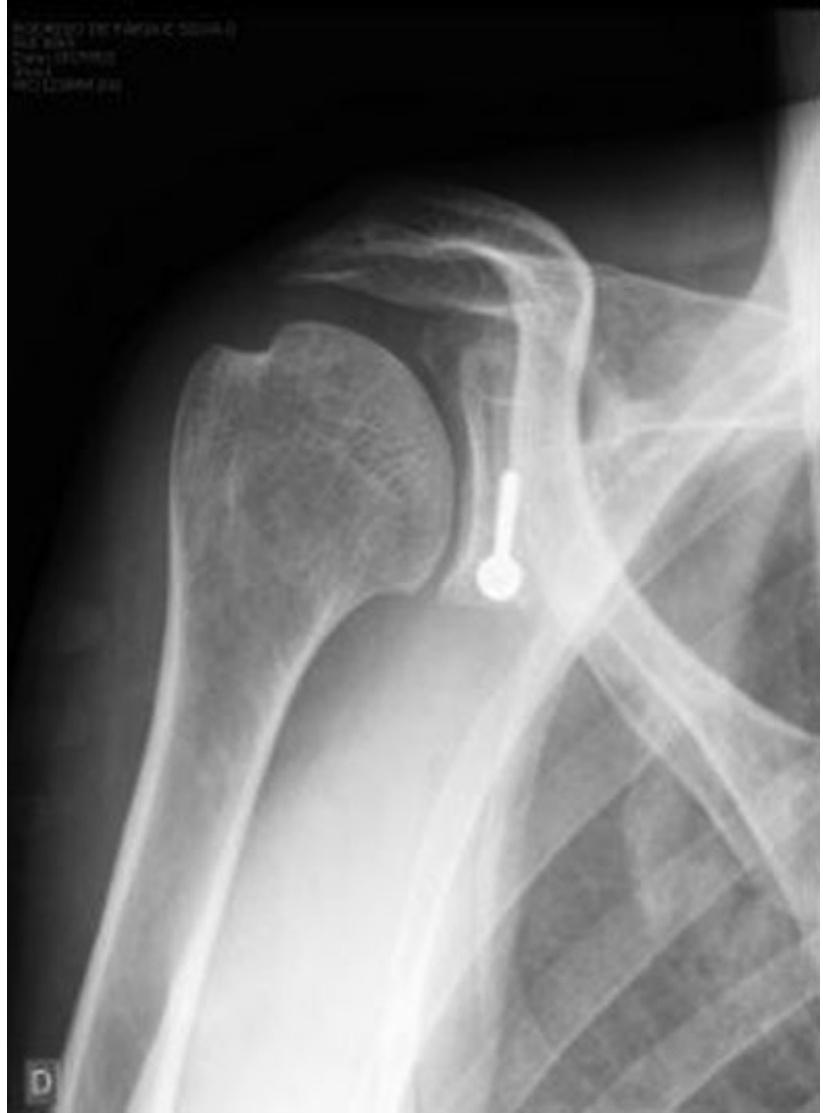

Fig. 3 Raio $x(R x)$ em incidência anteroposterior (AP) do ombro. Não se observa artrose. Enxerto consolidado e parafuso bem posicionado.

Latarjet pelo mesmo cirurgião entre 1990 e 1997. Desses pacientes, 26 (96\%) estavam satisfeitos, e apenas 1 (4\%) afirmou não estar completamente satisfeito, não havendo alteração em relação ao primeiro estudo. ${ }^{21}$

A abertura do subescapular juntamente com cápsula em forma de "L" foi fundamental para exposição do colo da glenoide, não influenciando no resultado final da rotação externa, permitindo a colocação correta do enxerto de coracoide fixado com um parafuso paralelo às articulações.
Isso foi considerado de fundamental importância, segundo Guiotti Filho et al. ${ }^{21}$ para os resultados conclusivos.

$\mathrm{Na}$ avaliação objetiva dos movimentos, houve recuperação da elevação e da rotação externa do ombro na maioria dos pacientes $(22 ; 84 \%)$. A restrição da rotação externa $>10^{\circ}$ foi encontrada nos casos de artrose moderada, e, nos casos de artrose grave, a restrição foi $>15^{\circ}$. Embora a restrição da rotação externa possa ter influência sobre a artropatia, ela não foi relevante em nossa avaliação.

Dos 27 pacientes avaliados, somente 3 tinham o sinal da apreensão positivo. Desses, 2 tiveram convulsões sem luxação, e 1 teve trauma com elevação e rotação externa do ombro, surgindo luxação que, após redução, foi manejada conservadoramente, sem necessidade de nova cirurgia.

Na avaliação radiográfica pelas incidências de Bernageau e Patte ${ }^{24}$ e oblíqua apical ${ }^{25}$ (-Figura 6), não foram identificados sinais de não consolidação do enxerto de coracoide e falha do material de síntese, assim como foi observado no primeiro estudo. $^{21}$

Neste estudo, dedicamos atenção especial ao grau de artropatia, principalmente pelo tempo de evolução póscirúrgico. A nossa expectativa era encontrar uma incidência maior de artropatia, mas foram observados 17 (63\%) casos de ombros sem sinais de artropatia, 7 (26\%) de artropatia suave, 2 (7\%) de artropatia moderada, e 1 (4\%) de artropatia grave.

Gordins et al. ${ }^{22}$ em estudo de longo prazo, utilizaram a idade de 22 anos como um parâmetro para avaliar os pacientes que tiveram luxação abaixo e acima dessa idade, e concluíram que os pacientes que tiveram luxação com mais de 22 anos apresentaram um grau de artropatia maior, o que é difícil de explicar, segundo a conclusão do seu trabalho.

Quando tentamos correlacionar a idade com o grau de artropatia e com a restrição de rotação externa, observamos que 15 pacientes sofreram o seu primeiro episódio de luxação com mais de 22 anos, mas apenas 1 apresentou artropatia grave e restrição de rotação externa $>10^{\circ}$ associada, e, portanto, o presente trabalho não encontrou dados suficientes para afirmar que a idade acima de 22 anos na primeira luxação pode ser um fator determinante para a evolução da artrose. 
Tabela 3 Correlação da idade com o grau de artropatia e com a restrição da rotação externa

\begin{tabular}{|c|c|c|c|c|c|c|c|c|c|c|c|c|c|c|c|c|}
\hline \multirow[t]{3}{*}{ Idade da primeira luxação } & \multicolumn{8}{|c|}{ Grau de artropatia } & \multicolumn{8}{|c|}{ Restrição de rotação $>10^{\circ}$} \\
\hline & \multicolumn{2}{|c|}{ Normal } & \multicolumn{2}{|c|}{ Suave } & \multicolumn{2}{|c|}{$\begin{array}{l}\text { Modera- } \\
\text { da }\end{array}$} & \multicolumn{2}{|c|}{ Grave } & \multicolumn{2}{|c|}{ Normal } & \multicolumn{2}{|c|}{ Suave } & \multicolumn{2}{|c|}{$\begin{array}{l}\text { Modera- } \\
\text { da }\end{array}$} & \multicolumn{2}{|c|}{ Grave } \\
\hline & E1 & $(\mathrm{E} 2)$ & E1 & (E2) & E1 & (E2) & E1 & $(\mathrm{E} 2)$ & E1 & $(\mathrm{E} 2)$ & E1 & (E2) & E1 & $(\mathrm{E} 2)$ & E1 & (E2) \\
\hline$\leq 22$ anos & 6 & 5 & 4 & 5 & 2 & 2 & 0 & 0 & 0 & 0 & 0 & 0 & 2 & 2 & 0 & 0 \\
\hline$>22$ anos & 11 & 9 & 3 & 4 & 0 & 1 & 1 & 1 & 1 & 1 & 1 & 1 & 1 & 1 & 1 & 1 \\
\hline
\end{tabular}

Abreviaturas: E1, examinador 1; E2, examinador 2.

Tabela 4 Medidas subjetivas e objetivas dos movimentos

\begin{tabular}{|l|l|l|}
\hline Classe & Testes & Médias \\
\hline \multirow{3}{*}{ Objetivas } & Rotação externa $\left(^{\circ}\right)$ & 83,8 \\
\cline { 2 - 3 } & Elevação $\left(^{\circ}\right)$ & 180 \\
\cline { 2 - 3 } & Rotação interna em extensão $(\mathrm{cm})$ & 1,8 \\
\hline \multirow{3}{*}{ Subjetivas } & Rotação externa $\left(^{\circ}\right)$ & 77,6 \\
\cline { 2 - 3 } & Elevação $\left(^{\circ}\right)$ & 180 \\
\cline { 2 - 3 } & Rotação interna em extensão $(\mathrm{cm})$ & 2,3 \\
\hline
\end{tabular}

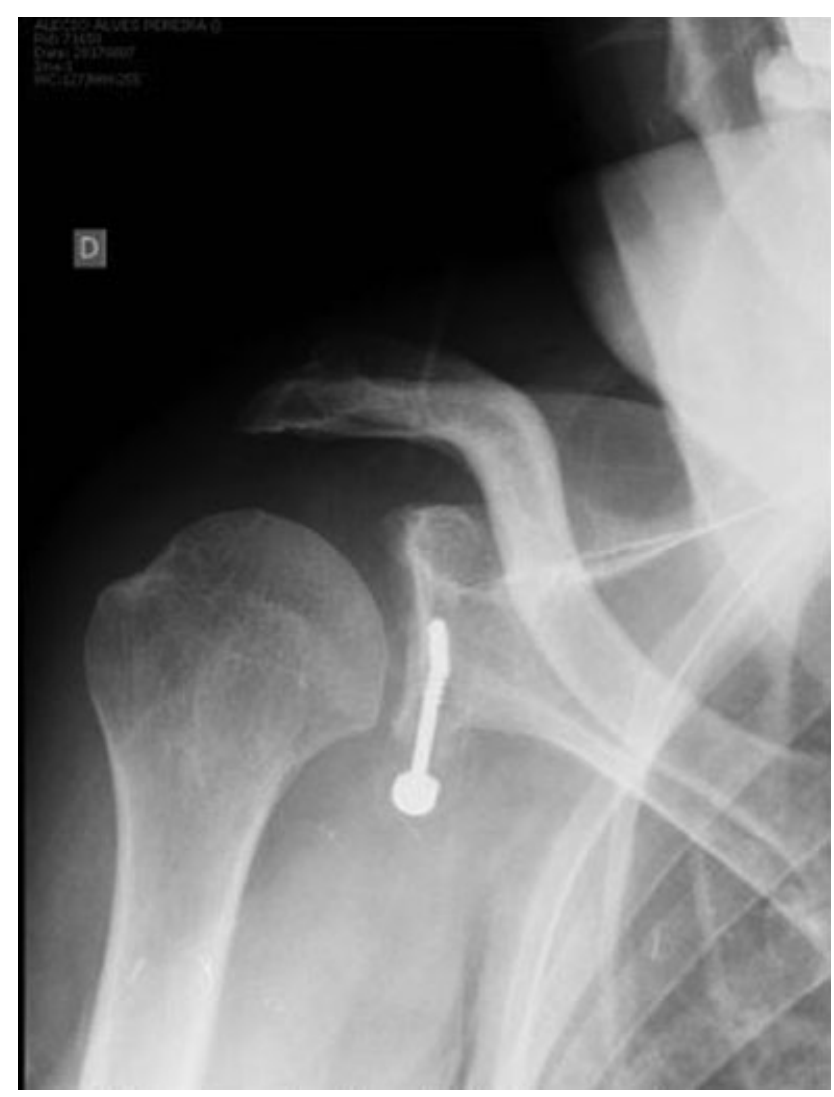

Fig. 4 Raio $x$ em incidência AP do ombro. Observa-se a quebra do parafuso, mas o enxerto está consolidado, sem sinais de artrose.

Gordins et al. ${ }^{22}$ relataram que seus números de artropatia na luxação anterior do ombro são, possivelmente, inferiores aos que poderiam ser esperados, e destacam que a artropatia vista das incidências AP e axial, relacionadas à luxação

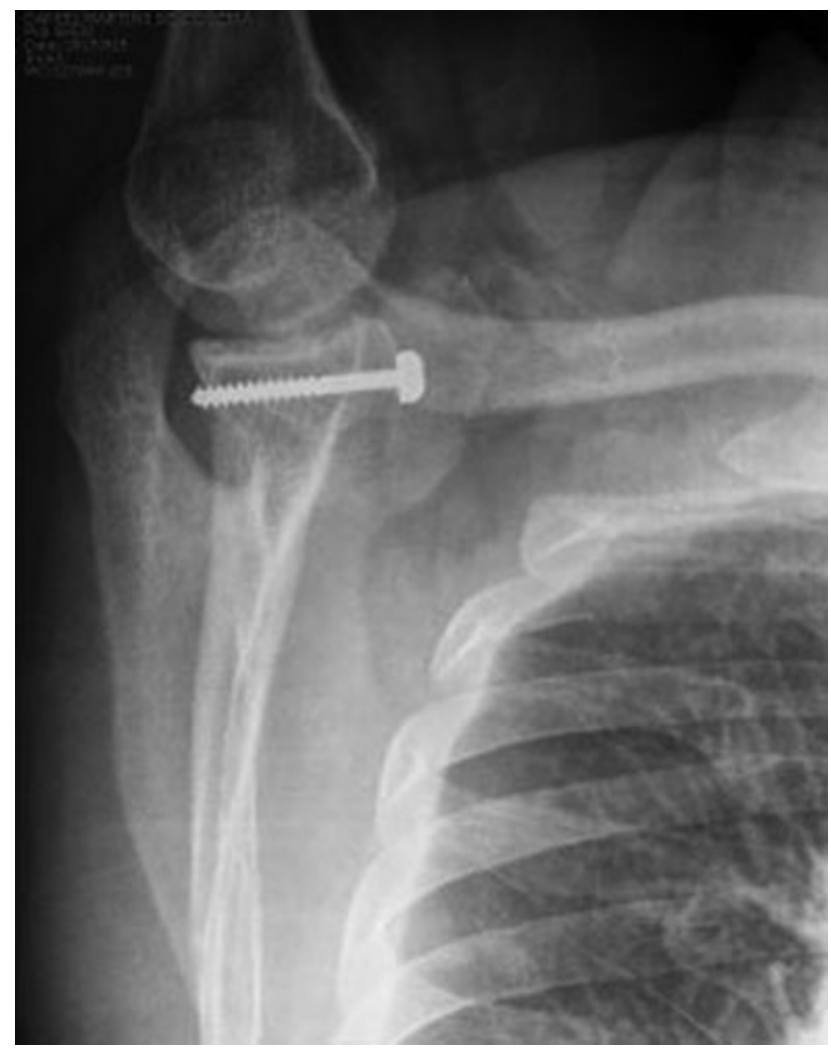

Fig. 5 Raio $x$ do ombro em incidência de Bernageau e Patte. ${ }^{24}$ Observam-se o comprimento do parafuso, a posição paralela à glenoide, e o enxerto consolidado.

anterior do ombro, é uma parte da história natural desta condição.

Isto põe em questão se o procedimento cirúrgico é o principal fator etiológico da artropatia. ${ }^{26}$ Além disso, não foi possível encontrar qualquer associação entre o grau de perda de rotação externa após 2 anos e a artropatia aos 15 anos. Esses achados estão de acordo com as observações de Van der Zwaag et al. ${ }^{27}$ que também não encontraram correlação entre a rotação externa 6 meses após a cirurgia e o desenvolvimento de artrose glenoumeral de 10 a 40 anos depois.

Segundo Gordins et al. ${ }^{22}$ o grau de artropatia após o procedimento de Bristow-Latarjet parece seguir a história natural da instabilidade do ombro com relação à degeneração artropática comum com o tempo. A restrição pós-operatória da rotação externa não aumenta a artropatia posterior. A classificação da artropatia varia segundo os 
Tabela 5 Resultados do raio x nas incidências de Bernageau e Patte $^{24}$ e oblíqua apical

\begin{tabular}{|l|l|l|l|}
\hline Quesitos & Examinador 1 & Examinador 2 & Média \\
\hline \multicolumn{4}{|l|}{ Houve consolidação do enxerto ósseo? } \\
\hline Sim & 27 & 27 & 27 \\
\hline Não & 0 & 0 & 0 \\
\hline Posicionamento adequado dos parafusos? & 27 \\
\hline Sim & 27 & 27 & 0 \\
\hline Não & 0 & 0 & \\
\hline Posicionamento adequado dos enxertos? & 26 \\
\hline Sim & 26 & 26 & 1 \\
\hline Não & 1 & 1 & 0 \\
\hline Há sinais de soltura do material de síntese? & 27 \\
\hline Sim & 0 & 0 & 27 \\
\hline Não & 27 & 27 & \\
\hline
\end{tabular}

Tabela 6 Resultado da Escala de Rowe et al. ${ }^{17}$

\begin{tabular}{|l|l|l|}
\hline Critérios & $\begin{array}{l}\text { Média da } \\
\text { pontuação }\end{array}$ & $\begin{array}{l}\text { Escala de } \\
\text { Rowe et al. }{ }^{17}\end{array}$ \\
\hline Estabilidade & 49 & $0-50$ \\
\hline Movimento & 18 & $0-20$ \\
\hline Função & 28 & $0-30$ \\
\hline Total & 95 & 100 \\
\hline
\end{tabular}

observadores e as incidências radiológicas, e isso está de acordo com os estudos anteriores de Gordins et al. ${ }^{22}$

Sugere-se que novos estudos sejam realizados, sendo necessário um estudo comparativo do lado operado com o lado oposto para se poder fazer uma melhor avaliação do aparecimento e da evolução da artropatia. Somente assim poderemos concluir se a artropatia encontrada faz parte da história natural ou surge em função do procedimento cirúrgico.

Hovelius et al. ${ }^{28}$ relataram uma taxa de satisfação de 98\% 15 anos após o reparo de Bristow-Latarjet, e os resultados encontrados por eles nesse estudo foram melhores do que os do primeiro estudo realizado também por eles, de 2 a 5 anos após o reparo. Os autores concluíram também que as subluxações ocasionais não são incomuns após um procedimento de Bristow-Latarjet, mas têm pouca influência nos resultados globais em longo prazo. ${ }^{20,29,30}$ Observamos que é possível ocorrer melhora nos resultados em longo prazo, mas, em nossa avaliação, a subluxação não ocorreu.

No presente estudo, os resultados negativos constatados em 3 (11\%) pacientes podem estar correlacionados, em dois deles, com histórico de convulsão; e, no outro paciente, ocorreu luxação por um novo trauma, situação que mudou

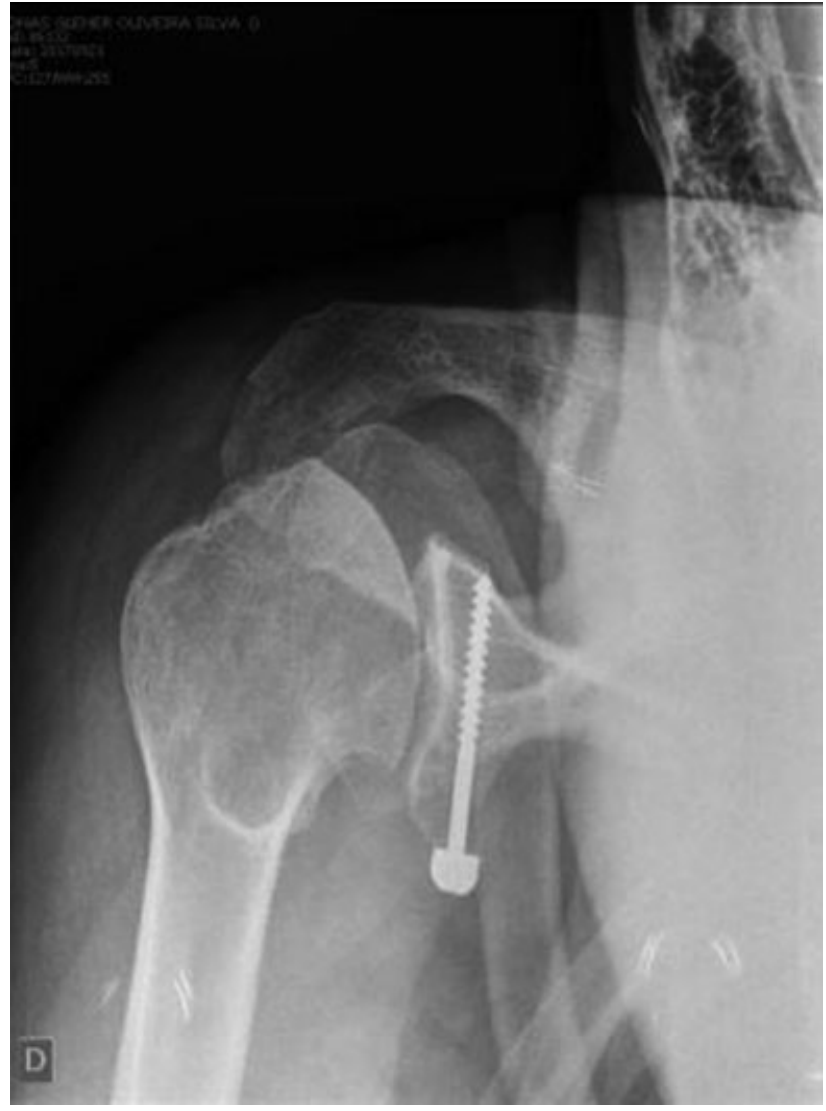

Fig. 6 Raio x em incidência oblíqua apical. Observam-se a posição correta e ideal do parafuso antingindo a cortical posterior, o enxerto consolidado, e o parafuso paralelo à superfície da glenoide.

o parafuso de posição. O paciente identificado com artrose grave e restrição grave da rotação externa apresentava lesão axonal do nervo axilar antes da cirurgia, e, apesar disso, não apresentou recidiva da luxação.

É importante destacar que, atualmente, a preferência é pela colocação do enxerto horizontal e fixado com dois parafusos paralelos, utilizando o ligamento coracoacromial para ser suturado na cápsula articular, com o objetivo permanecendo o mesmo.

\section{Conclusão}

Entre a primeira e a segunda avaliações, não houve alteração quanto à eficácia da técnica de Bristow-Latarjet. A observação cuidadosa dos critérios da técnica por nós relatada foi fundamental para evitar complicações. A ocorrência de artropatia em longo prazo não foi relevante em nossa avaliação. Pelas evidências do presente estudo, somente o procedimento cirúrgico não é a causa do surgimento da artropatia, mas a falha na sua execução.

\section{Conflito de Interesses}

Os autores declaram não haver conflito de interesses. 


\section{Referências}

1 Hovelius L. Incidence of shoulder dislocation in Sweden. Clin Orthop Relat Res 1982;(166):127-131

2 Helfet AJ. Coracoid transplantation for recurring dislocation of the shoulder. J Bone Joint Surg Br 1958;40(02):198-202

3 Latarjet M. A propos du traitement des luxations récidivantes de l'épaule. Lyon Chir 1954;49(08):994-997

4 Patte D, Bernageau J, Rodineau J, Gardes JC. [Unstable painful shoulders (author's transl)]. Rev Chir Orthop Repar Appar Mot 1980;66(03):157-165

5 May VR Jr. A modified Bristow operation for anterior recurrent dislocation of the shoulder. J Bone Joint Surg Am 1970;52(05): 1010-1016

6 Ferreira Filho AA. Tratamento de luxação anterior recidivante do ombro pela técnica Bristow-Latarjet [tese]. São Paulo: Faculdade de Medicina, Universidade de São Paulo; 1984

7 Young DC, Rockwood CA Jr. Complications of a failed Bristow procedure and their management. J Bone Joint Surg Am 1991;73 (07):969-981

8 Artz T, Huffer JM. A major complication of the modified Bristow procedure for recurrent dislocation of the shoulder. A case report. J Bone Joint Surg Am 1972;54(06):1293-1296

9 Bach BR Jr. Arthroscopic removal of painful Bristow hardware. Arthroscopy 1990;6(04):324-326

10 Bach BR Jr, O'Brien SJ, Warren RF, Leighton M. An unusual neurological complication of the Bristow procedure. A case report. J Bone Joint Surg Am 1988;70(03):458-460

11 Clancy MJ. False aneurysm of the axillary artery as a complication of the modified Bristow procedure. Injury 1987;18(06):427-428

12 Fee HJ, McAvoy JM, Dainko EA. Pseudoaneurysm of the axillary artery following a modified Bristow operation: report of a case and review. J Cardiovasc Surg (Torino) 1978;19(01):65-68

13 Iftikhar TB, Kaminski RS, Silva I Jr. Neurovascular complications of the modified Bristow procedure. A case report. J Bone Joint Surg Am 1984;66(06):951-952

14 Lower RF, McNiesh LM, Callaghan JJ. Computed tomographic documentation of intra-articular penetration of a screw after operations on the shoulder. A report of two cases. J Bone Joint Surg Am 1985;67(07):1120-1122

15 Nielson $A B$, Nielsen $K$. The modified Bristow procedure for recurrent anterior dislocation of the shoulder. Results and complications. Acta Orthop Scand 1982;53(02):229-232

16 Richards RR, Hudson AR, Bertoia JT, Urbaniak JR, Waddell JP. Injury to the brachial plexus during Putti-Platt and Bristow procedures. A report of eight cases. Am J Sports Med 1987;15 (04):374-380

17 Rowe CR, Zarins B, Ciullo JV. Recurrent anterior dislocation of the shoulder after surgical repair. Apparent causes of failure and treatment. J Bone Joint Surg Am 1984;66(02):159-168

18 Strömqvist B, Wingstrand H, Egund N. Recurrent shoulder dislocation and screw failure after the Bristow-Latarjet procedure. A case report. Arch Orthop Trauma Surg 1987;106(04):260-262

19 Zuckerman JD, Matsen FA 3rd. Complications about the glenohumeral joint related to the use of screws and staples. J Bone Joint Surg Am 1984;66(02):175-180

20 Weaver JK, Derkash RS. Don't forget the Bristow-Latarjet procedure. Clin Orthop Relat Res 1994;(308):102-110

21 Guiotti Filho J, Borges AC, Rabelo LW, Daher WR. Instabilidade anterior do ombro: tratamento cirúrgico pela técnica de BristowLaterjet. Rev Bras Ortop 1998;33(09):724-730

22 Gordins V, Hovelius L, Sandström B, Rahme H, Bergström U. Risk of arthropathy after the Bristow-Latarjet repair: a radiologic and clinical thirty-three to thirty-five years of follow-up of thirty-one shoulders. J Shoulder Elbow Surg 2015;24(05):691-699

23 Samilson RL, Prieto V. Dislocation arthropathy of the shoulder. J Bone Joint Surg Am 1983;65(04):456-460

24 Bernageau J, Patte D. Le profil glenoide. J Traumatol Sport 1984;1 (01):15-19

25 Garth WP Jr, Slappey CE, Ochs CW. Roentgenographic demonstration of instability of the shoulder: the apical oblique projection. A technical note. J Bone Joint Surg Am 1984;66(09):1450-1453

26 Hawkins RJ, Angelo RL. Osteoartrose Glenohumeral. A late complication of the Putti-Platt repair. J Bone Joint Surg Am 1990;72 (08):1193-1187

27 Van der Zwaag HM, Brand R, Obermann WR, Rozing PM. Osteoarthrosis glenohumeral after Plutti-Platt. J Shoulder Elbow Surg 1999;8(03):252-258

28 Hovelius L, Sandström B, Sundgren K, Saebö M. One hundred eighteen Bristow-Latarjet repairs for recurrent anterior dislocation of the shoulder prospectively followed for fifteen years: study Iclinical results. J Shoulder Elbow Surg 2004;13(05):509-516

29 Hill JA, Lombardo SJ, Kerlan RK, et al. The modification BristowHelfet procedure for recurrent anterior shoulder subluxations and dislocations. Am J Sports Med 1981;9(05):283-287

30 Uhorchak JM, Arciero RA, Huggard D, Taylor DC. Recurrent shoulder instability after open reconstruction in athletes involved in collision and contact sports. Am J Sports Med 2000;28(06):794-799 